The final journal version of this paper appears in A. Lastra, J. R. Sendra, L. X. C. Ngô and F. Winkler (2014). Rational General Solutions of Systems of Autonomous Ordinary Differential Equations of AlgebroGeometric Dimension One. Publ. Math. Debrecen Publ. Math. Debrecen 2015 / 86 / 1-2 49-69. DOI: 10.5486/PMD.2015.6032 and it is available at http://dx.doi.org/10.5486/PMD.2015.6032

\title{
Rational General Solutions of Systems of Autonomous Ordinary Differential Equations of Algebro-Geometric Dimension One
}

\author{
A. Lastra J. R. Sendra \\ Dpto. de Física y Matemáticas, \\ Universidad de Alcalá. Alcalá de Henares, Madrid, Spain \\ \{Alberto.Lastra,Rafael.Sendra\}@uah.es \\ L. X. C. Ngô \\ Dept. of Mathematics, \\ Quy Nhon University, Quy Nhon, Vietnam \\ ngolamxuanchau@qnu.edu.vn \\ F. Winkler \\ Research Inst. for Symbolic Computation (RISC) \\ Johannes Kepler Universität Linz, A-4040 Linz, Austria \\ Franz.Winkler@risc.jku.at
}

\begin{abstract}
An algebro-geometric method for determining the rational solvability of autonomous algebraic ordinary differential equations is extended from single equations of order 1 to systems of equations of arbitrary order but dimension 1 in the algebro-geometric sense. We provide necessary conditions, for the existence of rational solutions, on the degree and on the structure at infinity of the associated algebraic curve. Furthermore, from a rational parametrization of a planar projection of the corresponding space curve one deduces, either by derivation or by lifting the planar parametrization, the existence and actual computation of all rational solutions if they exist. Moreover, if the differential polynomials are defined over the rational numbers, we can express the rational solutions over the same field of coefficients.
\end{abstract}

Keywords: algebraic ordinary differential equations, rational solutions, parametrization of curves. 


\section{Introduction}

An algebraic ordinary differential equation (AODE) is a polynomial relation among a function, (finitely many of) their derivatives, and the variable of differentiation. If the variable of differentiation does not explicitly appear in this relation, the AODE is called autonomous. In case the relation polynomial is linear, i.e. for linear ordinary differential equations, we have well-known solution methods. However, no general solution method is available for non-linear AODEs.

Feng and Gao [3], [4] have presented an algorithm for deciding the existence of nontrivial rational solutions of autonomous AODEs of order 1. In a nutshell, they take the bi-variate polynomial $f$ defining the autonomous AODE, consider the algebraic curve it defines, and check whether this curve admits a rational parametrization; from such a rational parametrization they decide the existence of rational solutions of the given AODE and, in the affirmative case, compute a rational general solution. Their method makes critical use of degree bounds for rational parametrizations of algebraic curves, as developed in [10]. So from the known degree bound for the proper parametrizations of the corresponding curve we get a degree bound for rational general solutions. In [6] and [8] Ngô and Winkler have extended this decision and solution method to general, possibly non-autonomous, AODEs of order 1. Under suitable conditions also AODEs of higher order can be treated in a similar way as shown in [9]. On the other hand, in [2], an upper bound for the degree of the rational solutions of a first-order (non necessarily autonomous) differential equation is given. Therefore, by introducing undetermined coefficients one derives an algebraic system of equations whose solutions provide the rational solutions of the differential equation. Thus, it yields an alternative algorithm to those mentioned above.

In the present paper we go a step further and we consider systems of autonomous AODEs of arbitrary order $n$. Such a system is given by a set of $(n+1)$-variate polynomials, which define an algebraic variety in $(n+1)$-dimensional space. We assume that this variety to be of dimension 1, i.e., a curve. We show that Feng and Gao's theory for detecting the existence, and actual computation, of rational solutions of autonomous equations of order 1 can be extended to such systems. From a computational point of view, the problem is then solved by considering a suitable planar projection, of the space curve, to afterwards lift the information to the system. In fact, if the differential polynomials are defined over the rational numbers, we can express the rational solutions over the same field of coefficients. In addition to this, necessary conditions for the existence of rational solutions are related to the degree and to the structure at infinity of the associated algebraic curve. Alternatively, once the planar curve is computed, the bound in [2] can be applied to provide a candidate of solution that might be lifted to a solution of the system. In this paper, we focus on the extension of Feng and Gao's approach.

The computations in this paper have been done with the mathematical software 
Maple.

\section{Notation, Basic Assumptions and First Results}

Throughout this paper, we will use the following notation. $\mathbb{Q}$ is the field of rational numbers and $\overline{\mathbb{Q}}$ is its algebraic closure. Let

$$
\mathcal{F}=\left\{F_{j}(\bar{w})\right\}_{j \in J \subset \mathbb{N}} \subset \mathbb{Q}[\bar{w}],
$$

where $\bar{w}=\left(w_{0}, w_{1}, \ldots, w_{n}\right)$, be a finite set of polynomials in $\mathbb{Q}[\bar{w}]$. All the results in this paper are also valid if we replace $\mathbb{Q}$ by a computable field extension of $\mathbb{Q}$ as, for instance, $\mathbb{Q}(\sqrt[3]{2})$ or $\mathbb{Q}(\sqrt{-1})$. However, for simplicity in the exposition we develop the theory taking $\mathbb{Q}$ as the ground field. In addition, we assume that

(i) for all $j \in J$, there exists $i \in\{1, \ldots, n\}$ such that $\operatorname{deg}_{w_{i}}\left(F_{j}\right)>0$,

(ii) the algebraic variety defined by $\mathcal{F}$ in $\overline{\mathbb{Q}}^{n+1}$, the $(n+1)$-dimensional space over $\overline{\mathbb{Q}}$, is a rational curve. We denote this curve by $\mathcal{C}$. Furthermore, we assume that

$$
\mathcal{R}(t)=\left(r_{1}(t), \ldots, r_{n+1}(t)\right)
$$

is a proper rational parametrization of $\mathcal{C}$ with coefficients in a field $\mathbb{F}$ such that $\mathbb{Q} \subset \mathbb{F} \subset \overline{\mathbb{Q}}$. We can assume that $\mathbb{F}$ is optimal, and hence (see Chapter 5 in $[11]) \mathbb{F}$ is an algebraic field extension of $\mathbb{Q}$ of degree at most 2. Recall that a proper parametrization is a birational mapping from the affine line to the curve; or, equivalently $\mathbb{F}(\mathcal{R}(t))=\mathbb{F}(t)$; see Section 4.2 and 6.1 in [11] for further details. We also assume that $r_{1}(t)$ is not constant; see below that this condition does not imply any loss of generality.

Rational parametrizations are assumed to be expressed in reduced formed; i.e., with relatively prime numerator and denominator.

Associated to $\mathcal{F}$ we consider the autonomous algebraic system $\mathcal{S}$ of ordinary differential equations (system of AODEs)

$$
\mathcal{S}=\left\{F_{j}\left(y, y^{\prime}, \ldots, y^{(n)}\right)=0\right\}_{j \in J}
$$

where $y$ is an indeterminate over a differential extension field of $\overline{\mathbb{Q}}(x)$, and ' denotes the differentiation w.r.t. $x$. Such a system, associated to an $\mathcal{F}$ defining a space curve, is called a system of AODEs of algebro-geometric dimension 1; observe that this notion of dimension is not the usual concept of dimension in differential algebra.

Note that, because of condition (i) on $\mathcal{F}$, all equations in $\mathcal{S}$ involve at least one

derivative $y^{(i)}$. We recall that a function $f(x)$, which is $n$ times differentiable, is a (nontrivial) solution of $\mathcal{S}$ if it is not constant and

$$
F_{i}\left(f(x), f^{\prime}(x), f^{\prime \prime}(x), \ldots, f^{(n)}(x)\right)=0, \quad \forall i \in J
$$


If $f(x) \in \overline{\mathbb{Q}}(x) \backslash \mathbb{Q}$ we say that $f$ is a (nontrivial) rational solution. Let $f(x)$ be a rational solution (and hence non-constant) of $\mathcal{S}$, then we consider the rational parametrization

$$
\mathcal{P}_{f}(t)=\left(f(t), f^{\prime}(t), \ldots, f^{(n)}(t)\right)
$$

that we call the integral parametrization generated by $f(x)$. Obviously, every integral parametrization parametrizes $\mathcal{C}$. Since our goal is to study the existence of nontrivial rational solutions of $\mathcal{S}$, the condition in (ii) on $r_{1}(t)$ is now a clear requirement; note that if a rational parametrization of $\mathcal{C}$ has its first component constant, then any other rational parametrization of $\mathcal{C}$ has the property.

The following lemma is a direct consequence of Theorem 2 in [3] or Theorem 3.7 in [4].

Lemma 2.1. Let $f \in \overline{\mathbb{Q}}(t)$ be non-constant. Then $\overline{\mathbb{Q}}\left(f(t), f^{\prime}(t), \ldots, f^{(n)}(t)\right)=\overline{\mathbb{Q}}(t)$.

Proof. W.l.o.g. we assume that $f$ is in reduced form. We apply induction on $n$. For $n=1$ the result follows by Theorem 2 in [3]. Let the result be true up to $n-1$. Then,

$$
\begin{gathered}
\overline{\mathbb{Q}}\left(f(x), f^{\prime}(x), f^{\prime \prime}(x), \ldots, f^{(n)}(x)\right)=\overline{\mathbb{Q}}\left(f(x), f^{\prime}(x), \ldots, f^{(n-1)}(x)\right)\left(f^{(n)}(x)\right) \\
=\overline{\mathbb{Q}}(x)\left(f^{(n)}(x)\right)=\overline{\mathbb{Q}}\left(x, f^{(n)}(x)\right)=\overline{\mathbb{Q}}(x) .
\end{gathered}
$$

Corollary 2.2. Every integral parametrization is a proper parametrization of $\mathcal{C}$.

Our goal is to study the existence of rational solutions of $\mathcal{S}$ and, in the positive case, to determine a general rational solution. We say that a rational function $R(x, C) \in$ $\overline{\mathbb{Q}}(x, C)$ is a general rational solution of $\mathcal{S}$ if for every non-constant rational solution $f(x)$ of $\mathcal{S}$ there exists $C_{0} \in \overline{\mathbb{Q}}$ such that $f(x)=R\left(x, C_{0}\right)$. The next theorem shows that a general rational solution can be derived from any particular (non-constant) rational solution.

Theorem 2.3. Let $f(x)$ be a non-constant rational solution of $\mathcal{S}$, then $f(x+C)$ is a general rational solution of $\mathcal{S}$.

Proof. Let $g(x)$ be a non-constant rational solution of $\mathcal{S}$. Then, by Corollary 2.2, $\mathcal{P}_{f}(t)$ and $\mathcal{P}_{g}(t)$ are proper parametrizations of $\mathcal{C}$. Therefore, by Lüroth's theorem (see, e.g. Section 6.1. in [11]), there exists $h(t)=\frac{a t+b}{c t+d} \in \overline{\mathbb{Q}}(t), a d-b c \neq 0$, such that $\mathcal{P}_{g}(t)=\mathcal{P}_{f}(h(t))$. In particular, differentiating in the first component and taking into account the second components, $g^{\prime}(t)=f^{\prime}(h(t)) h^{\prime}(t)=f^{\prime}(h(t))$. Therefore, since $f(x)$ is not constant, $f^{\prime}(h) \neq 0$ and hence $h^{\prime}(t)=1$. Thus, $h(t)=t+C_{0}$, for some $C_{0} \in \overline{\mathbb{Q}}$.

Some of the reasonings in this paper take into account whether a solution of a system is polynomial or not. The polynomial nature only depends on the system. Indeed, one has the next result that follows directly from Theorem 2.3. 
Corollary 2.4. The following statements are equivalent

1. $\mathcal{S}$ has a non-constant polynomial solution.

2. All non-constant rational solutions of $\mathcal{S}$ are polynomial.

\section{Partial Degree of Space Curves}

In this section we introduce the notion of partial degree of a space curve, and we see how to compute it from a rational parametrization when the curve is rational. Let $\mathcal{D} \subset \overline{\mathbb{Q}}^{m}$ be an irreducible affine algebraic curve in the $m$-dimensional affine space $\overline{\mathbb{Q}}^{m}$.

We recall that the degree of $\mathcal{D}$ is defined as the number of intersection points of $\mathcal{D}$ with a generic hyperplane in $\overline{\mathbb{Q}}^{m}$ (see Def. 18.1 in [5]); we denote it by $\operatorname{deg}(\mathcal{D})$. Motivated by this notion, we introduce the notion of partial degree. For $i \in\{1, \ldots, m\}$,

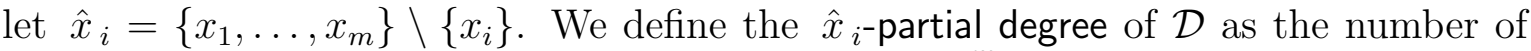
intersection points of $\mathcal{D}$ with a generic hyperplane in $\overline{\mathbb{Q}}^{m}$ of the form $x_{i}=a$; we denote it by $\operatorname{deg}_{\hat{x}_{i}}(\mathcal{D})$. The following lemma ensures that the partial degree notion is well defined.

Lemma 3.1. Let $\mathcal{D}$ be an irreducible algebraic curve in $\overline{\mathbb{Q}}^{m}$. Let $i \in\{1, \ldots, m\}$. For all but finitely many values $a \in \overline{\mathbb{Q}}$ the intersection of $\mathcal{D}$ with the hyperplane $x_{i}=a$ is either always empty or always finite. Moreover, in the second case, for all but a finite number of exceptions of these hyperplanes, the number of intersection points is invariant.

Proof. W.l.o.g. we assume that $i=1$. Moreover, for $a \in \overline{\mathbb{Q}}$, we denote by $\mathcal{H}_{a}$ the hyperplane defined by $x_{1}=a$. We observe that $\operatorname{card}\left(\mathcal{D} \cap \mathcal{H}_{a}\right)=\infty$ if and only if $\mathcal{D} \subset \mathcal{H}_{a}$. Therefore, in that case $\mathcal{D} \cap \mathcal{H}_{b}=\emptyset$ for $b \neq a$. So, let us assume that there does not exist $a \in \overline{\mathbb{Q}}$ such that $\mathcal{D} \subset \mathcal{H}_{a}$. In this situation, $\operatorname{card}\left(\mathcal{D} \cap \mathcal{H}_{a}\right)<\infty$ for all $a \in \overline{\mathbb{Q}}$. Let us see that for almost all $a \in \overline{\mathbb{Q}}$, this cardinality is invariant.

Let $\mathrm{I} \subset \overline{\mathbb{Q}}[\bar{x}]$, with $\bar{x}=\left(x_{1}, \ldots, x_{m}\right)$, be the ideal of $\mathcal{D}$, and $\mathcal{F}\left(x_{1}, \ldots, x_{m}\right)$ a finite set of generators of I. I is 1-dimensional but, for almost all $a \in \overline{\mathbb{Q}}$, the ideal $\mathrm{I}_{a}$ generated by $\mathcal{F}\left(a, x_{2}, \ldots, x_{m}\right)$ in $\mathbb{K}\left[x_{2}, \ldots, x_{m}\right]$ is 0 -dimensional, since it corresponds to $\mathcal{D} \cap \mathcal{H}_{a}$. Now, let $\mathcal{G}(\bar{x})$ be a reduced Gröbner basis of I w.r.t. the lex order, with $x_{m}>\cdots>x_{1}$; reduced in the sense of Def. 5 in [1], pg. 90. On the other hand, using ex. 7, pg. 283, in [1], for all $a \in \overline{\mathbb{Q}}$, but finitely many exceptions, it holds that $\mathcal{G}\left(a, x_{2}, \ldots, x_{m}\right)$ is a Gröbner basis of $\mathrm{I}_{a}$. Therefore, $\mathcal{G}(\bar{x})$ has to be of the form

$$
\left\{g_{1,1}\left(x_{1}, x_{2}\right), g_{2,1}\left(x_{1}, x_{2}, x_{3}\right), \ldots, g_{2, k_{2}}\left(x_{1}, x_{2}, x_{3}\right), \ldots, g_{m, 1}(\bar{x}), \ldots, g_{m, k_{m}}(\bar{x})\right\} .
$$

Now, let $\mathbb{F}_{1}$ be the algebraic closure of $\overline{\mathbb{Q}}\left(x_{1}\right)$. We see $g_{1,1}$ as polynomial in $\mathbb{F}_{1}\left[x_{2}, \ldots, x_{m}\right]$. Then, the number of different roots of $g_{1,1}\left(x_{2}\right) \in \mathbb{F}_{1}\left[x_{2}\right]$ is fixed; say $\ell_{1}$. We assume w.l.o.g. that $g_{1,1}$ is irreducible over $\overline{\mathbb{Q}}\left(x_{1}\right)$; otherwise we proceed with each irreducible factor. Next we consider the algebraic closure $\mathbb{F}_{2}$ of the quotient field 
of $\mathbb{K}\left(x_{1}\right)\left[x_{2}\right] /\left\langle g_{1,1}\left(x_{2}\right)\right\rangle$, and we see the polynomials in $g_{2, j}$ as polynomials in $\mathbb{F}_{2}\left[x_{3}\right]$. Then, since the system has solutions, $\operatorname{gcd}_{\mathbb{F}_{2}\left[x_{3}\right]}\left(g_{2,1}, \ldots, g_{2, k_{2}}\right)$ is not constant, let $\ell_{2}$ be the number of different roots of this gcd. Extending this argument we get that the number of solution of the $\mathcal{G}\left(a, x_{2}, \ldots, x_{m}\right)$, for a generic in $\overline{\mathbb{Q}}$, is $\ell_{1} \cdots \ell_{m}$.

The following theorem generalizes Theorem 4.21 in [11].

Theorem 3.2. Let $\mathcal{D}$ be rational and let

$$
\mathcal{P}(t)=\left(\frac{p_{1}(t)}{q_{1}(t)}, \ldots, \frac{p_{m}(t)}{q_{m}(t)}\right)
$$

be a proper rational parametrization of $\mathcal{D}$ in reduced form. For $i \in\{1, \ldots, m\}$, it holds that

$$
\operatorname{deg}_{\hat{x}_{i}}(\mathcal{D})=\operatorname{deg}_{t}\left(\frac{p_{i}(t)}{q_{i}(t)}\right)
$$

Proof. We assume w.l.o.g. that $i=m$. If $p_{m} / q_{m}=\lambda \in \overline{\mathbb{Q}}$ then, $\mathcal{D}$ is included in the plane $x_{m}=\lambda$, and hence the intersection of $\mathcal{D}$ with a generic plane of the form $x_{m}=a$ is empty, which agrees with the degree of $m$-component of $\mathcal{P}(t)$. So, let us assume that $p_{m} / q_{m}$ is not constant. We consider the subset $\Delta$ of $\overline{\mathbb{Q}}$ of those elements $z$ satisfying that

1. $z$ is the $m$-coordinate of a point in $\mathcal{D} \backslash \mathcal{P}(\overline{\mathbb{Q}})$.

2. $z$ is the $m$-coordinate of a point in $\mathcal{D}$ reachable by $\mathcal{P}(t)$ by exactly one parameter value.

3. the number of intersection points of $\mathcal{D}$ with the hyperplane $x_{m}=z$ is smaller than $\operatorname{deg}_{\hat{x}_{m}}(\mathcal{D})$

4. $p_{m}(t)-q_{m}(t) z$ is not square-free.

5. $\operatorname{deg}\left(p_{m}(t)-q_{m}(t) z\right)<\max \left\{\operatorname{deg}\left(p_{m}\right), \operatorname{deg}\left(q_{m}\right)\right\}$.

Let us assume that $\Delta$ is either empty or finite. Indeed, by the definition of parametrization $\operatorname{card}(\mathcal{D} \backslash \mathcal{P}(\overline{\mathbb{Q}}))<\infty$, by definition of properness the set of values not satisfying (2) is also finite, for (3) one uses Lemma 3.1, the reason for (4) comes from Lemma 4.19 in [11], and (5) cannot happen, at most, for a value of $z$.

Now, let $a \in \overline{\mathbb{Q}} \backslash \Delta$, let $\mathcal{H}_{a}$ be the hyperplane defined by $x_{m}=a$, and $f(t)=$ $p_{m}(t)-q_{m}(t) a$. Clearly, by $(3)$ and $(4), \operatorname{deg}(f) \leq \operatorname{deg}_{\hat{x}_{m}}(\mathcal{D})$. Because of $(1)$ and $(2)$, all points in $\mathcal{D} \cap \mathcal{H}_{a}$ are reachable by $\mathcal{P}(t)$ exactly once. So, by $(3), \operatorname{deg}(f) \geq \operatorname{deg}_{\hat{x}_{m}}(\mathcal{D})$. Therefore, $\operatorname{deg}(f)=\operatorname{deg}_{\hat{x}_{m}}(\mathcal{D})$. Now, by $(4), \operatorname{deg}(f)=\operatorname{deg}\left(p_{m} / q_{m}\right)$. 


\section{Existence of Rational Solutions}

We start this analysis with a generalization to systems of AODEs of the necessary condition given in Theorem 3 in [3].

Theorem 4.1. Let $\mathcal{S}$ admit a non-constant rational solution. Then, for $i=$ $0,1,2, \ldots, n-1$, we have

$$
\operatorname{deg}_{\left\{w_{0}, w_{1}, \ldots, w_{n}\right\} \backslash\left\{w_{i}\right\}}(\mathcal{C})-1 \leq \operatorname{deg}_{\left\{w_{0}, w_{1}, \ldots, w_{n}\right\} \backslash\left\{w_{i+1}\right\}}(\mathcal{C}) \leq 2 \operatorname{deg}_{\left\{w_{0}, w_{1}, \ldots, w_{n}\right\} \backslash\left\{w_{i}\right\}}(\mathcal{C}) .
$$

Proof. Let $f(x)$ be a non-constant rational solution of the system $\mathcal{S}$. By Corollary 2.2, $\mathcal{P}_{f}(t)$ is a proper parametrization of $\mathcal{C}$. Let $i \in\{0,1,2, \ldots, n\}$. Theorem 3.2 and Lemma 7 in [3] lead us to the conclusion when considering $f^{(j)}(t)$, for $j=0,1, \ldots, n-1$.

The next result characterizes the existence, and actual description, of rational solutions of $\mathcal{S}$. The theorem is a generalization of Theorem 5 in [3], and the proof essentially follows the ideas described in [3].

Theorem 4.2. Let $\mathcal{R}(t)=\left(r_{1}(t), r_{2}(t), \ldots, r_{n+1}(t)\right) \in \overline{\mathbb{Q}}(t)^{n+1}$ be a proper rational parametrization of $\mathcal{C}$. The following assertions are equivalent:

(1) $\mathcal{S}$ admits a non-constant rational solution.

(2) There exist $a, b \in \overline{\mathbb{Q}}, a \neq 0$ such that either

(2.1) $a r_{j}^{\prime}(t)=r_{j+1}(t)$ for every $j=1, \ldots, n$, or

(2.2) $a(t-b)^{2} r_{j}^{\prime}(t)=r_{j+1}(t)$ for every $j=1, \ldots, n$.

Moreover, if one of these equivalent statements holds, then $f(x)=r_{1}(a x)$ (if (2.1) holds) and $f(x)=r_{1}\left(\frac{a b x-1}{a x}\right)$ (if (2.2) holds) is a non-constant rational solution of $\mathcal{S}$.

Proof. Let us assume there exists a non-constant rational solution $f(x)$ of $\mathcal{S}$. From Corollary 2.2, $\mathcal{P}_{f}(t)$ is a proper rational parametrization of $\mathcal{C}$ and by Lüroth's theorem, there exists $h(t)=\frac{c_{1} t+c_{2}}{c_{3} t+c_{4}} \in \overline{\mathbb{Q}}(t)$ with $c_{1} c_{4}-c_{2} c_{3} \neq 0$ such that $\mathcal{P}_{f}(t)=\mathcal{R}(h(t))$. Therefore,

$$
f^{(j)}(t)=r_{j+1}(h(t)), \quad j=0,1, \ldots, n .
$$

Let $j \in\{0,1, \ldots, n\}$. From (5) one obtains

$$
f^{(j)}(t)=\left(r_{j}(h(t))\right)^{\prime}=r_{j}^{\prime}(h(t)) h^{\prime}(t)=r_{j+1}(h(t)) .
$$

From here, the proof follows as in [3]. The converse implication follows directly.

The next corollaries follow from the previous theorem.

Corollary 4.3. Let $\mathcal{S}$ have non-constant rational solutions. It holds that 
1. Every proper rational parametrization of $\mathcal{C}$ provides a non-constant rational solution of $\mathcal{S}$.

2. If a proper parametrization of $\mathcal{C}$ has its coefficients in a field extension $\mathbb{L}$ of $\mathbb{Q}$, then the rational solution generated by this parametrization has its coefficients in $\mathbb{L}$.

Proof. Let $\mathcal{R}(t)=\left(r_{1}(t), \ldots, r_{n+1}(t)\right)$ be a proper parametrization of $\mathcal{C}$. Since, $\mathcal{S}$ has non-constant rational solutions, by Theorem 4.2 , there exist $a, b \in \overline{\mathbb{Q}}$ such that either $r_{1}(a x)$ or $r_{1}\left(\frac{a b x-1}{a x}\right)$ is a non-constant rational solution of $\mathcal{S}$. For proving $(2)$, let $\mathcal{R}(t)$ be over $\mathbb{L}$, and let $f(x)$ be the rational solution generated by $\mathcal{R}(t)$. Thus, by Theorem 4.2, $f(x)=r_{1}(a x)$ or $f(x)=r_{1}\left(\frac{a b x-1}{a x}\right)$, where $a, b \in \overline{\mathbb{Q}}$. We observe that $r_{1}^{\prime} \neq 0$ (see assumption (ii) in Section 2). Therefore, $\frac{r_{2}}{r_{1}^{\prime}}$ is well-defined and $\frac{r_{2}}{r_{1}^{\prime}} \in \mathbb{L}(t)$. Now taking into account statements (2.1) and (2.2) in Theorem 4.2, one has that either $a \in \mathbb{L}(t)$ or $a(t-b)^{2} \in \mathbb{L}(t)$. So, $a, b \in \mathbb{L}$, and hence $f(x) \in \mathbb{L}(x)$.

Corollary 4.4. Let $\mathcal{R}(t)=\left(r_{1}(t), r_{2}(t), \ldots, r_{n+1}(t)\right) \in \overline{\mathbb{Q}}(t)^{n+1}$ be a proper rational parametrization of $\mathcal{C}$. If there exists $j>1$ such that $r_{j}(t)$ has at least two different simple poles, then $\mathcal{S}$ has no rational solution.

Proof. Let us assume that $\mathcal{S}$ has a nontrivial rational solution. Then, by Theorem 4.2 either $r_{j}(t)$ or $\frac{1}{(t-b)^{2}} r_{j}(t)$, for some $b$, has a rational integral. However, both rational functions have at least a simple pole. Therefore, their integrals have a logarithmic part, and hence they cannot be rational functions (see formula (4.6.4) and Theorem 4.6.3. in [12]) which is a contradiction.

Corollary 4.5. Let $\mathcal{C}$ have degree higher than 1. If $\mathcal{C}$ has, at least, two simple points at infinity, and their multiplicities of intersection with the hyperplane at infinity is 1 , then $\mathcal{S}$ has no rational solutions.

Proof. Let $\mathcal{R}(t)=\left(r_{1}(t), \ldots, r_{n+1}(t)\right)$ be a proper affine rational parametrization of $\mathcal{C}$ with common denominator and such that its projectivization reaches the two simple points at infinity mentioned at the statement. Then, the common denominator of $\mathcal{R}(t)$ has two different simple roots. Now the result follows from Corollary 4.4.

Corollary 4.6. Let $F\left(y, y^{\prime}\right)=0$ be an irreducible autonomous first order algebraic differential equation. Let $F\left(w_{0}, w_{1}\right)$ be expressed as

$$
F\left(w_{0}, w_{1}\right)=f_{\ell}\left(w_{0}, w_{1}\right)+f_{\ell-1}\left(w_{0}, w_{1}\right)+\cdots+f_{0},
$$

where $f_{i}$ is homogeneous of degree $i$, and $f_{\ell} \neq 0$. If $f_{\ell}$ has at least two different simple linear factors over $\overline{\mathbb{Q}}$, then $F\left(y, y^{\prime}\right)=0$ does not have rational solutions.

Corollary 4.7. Let $F\left(y, y^{\prime}\right)=0$ be an (irreducible) autonomous first order algebraic differential equation of degree $2 ;$ i.e. the curve associated to the equation is an irreducible conic. If $F\left(y, y^{\prime}\right)=0$ has rational solutions, then $F\left(w_{0}, w_{1}\right)$ defines a parabola. 
Proof. It follows from Corollary 4.5 and taking into account that circles, ellipses and hyperbolas have exactly two different points at infinity, and hence with multiplicity of intersection 1. Parabolas have one point at infinity and the line at infinity is tangent.

We now turn our attention at the coefficients of the rational solutions of $\mathcal{S}$. We start with a technical lemma.

Lemma 4.8. Given $f(t)=\frac{c_{1} t+c_{2}}{c_{3} t+c_{4}} \in \overline{\mathbb{Q}}(t), c_{1} c_{4}-c_{2} c_{3} \neq 0$,

1. There exist $a, b \in \overline{\mathbb{Q}}$ such that $f^{\prime}(t)=a(f(t)-b)^{2}$ if and only if $c_{3} \neq 0$.

2. There exists $a \in \overline{\mathbb{Q}}$ such that $f^{\prime}(t)=a$ if and only if $c_{3}=0$.

Proof. If $f(t)=\frac{c_{1}}{c_{4}} t+c_{2}$, then $f^{\prime}(t)=\frac{c_{1}}{c_{4}}$ is not equal to $a(f(t)-b)$ for any $a, b \in \overline{\mathbb{Q}}$. This proves the "if case" in (1) and the "only if case" in (2) For the converse in (1), it suffices to take $a=\frac{c_{3}^{2}}{c_{1} c_{4}-c_{2} c_{3}}$ and $b=\frac{c_{1}}{c_{3}}$. If $c_{3} \neq 0$, then $f^{\prime}(t)$ cannot be a constant, and the proof is complete.

Remark 4.9. It is worth remarking that (2.1) in Theorem 4.2 holds if and only if the non-constant rational solution of $\mathcal{S}$ is obtained after a linear polynomial reparametrization of $r_{1}$, whereas (2.2) holds if and only if the linear reparametrization is rational but not polynomial. Moreover, the constants $a, b$ (resp. $a$ ) appearing in (2.2) ( resp. (2.1)) in Theorem 4.2 are those provided in the corresponding case in Lemma 4.8 .

The next result is a generalization of Theorem 6 in [3] and Theorem 3.14 in [4], and it provides information on the coefficients appearing in a rational solution $\mathcal{S}$. Its proof follows essentially the reasoning in [3]. But there, some details on the polynomial case are left. So we give here all details.

Theorem 4.10. Let $\mathcal{S}$ have non-constant rational solutions. Then, there exist nonconstant rational solutions with coefficients in $\mathbb{Q}$.

Proof. Let $\mathcal{R}(t)$ be the parametrization of $\mathcal{C}$ introduced in Section 2. As mentioned there, as a consequence of Chapter 5 in [11], $\mathcal{R}(t)$ can be taken with coefficients in $\mathbb{Q}(\alpha)$, where $\alpha$ is an algebraic number over $\mathbb{Q}$ of degree at most 2. Let $f(x)$ be the nontrivial rational solution generated by $\mathcal{R}(t)$. By Corollary 4.3, $f(x) \in \mathbb{Q}(\alpha)(x)$. So, we assume w.l.o.g. that $[\mathbb{Q}(\alpha): \mathbb{Q}]=2$. Let $\bar{\alpha}$ be the conjugate of $\alpha$ and $\bar{f}(x)$ the conjugation of $f(x)$.

We first assume that $f(x)$ is a polynomial. Since $\mathcal{F} \subset \mathbb{Q}[\bar{w}], \bar{f}(x)$ is also a rational solution of $\mathcal{S}$. From Corollary 2.1, $\mathcal{P}_{f}(t)$ and $\mathcal{P}_{\bar{f}}(t)$ are proper parametrizations of $\mathcal{C}$. Moreover, by Lüroth's theorem, there exists $h(t) \in \overline{\mathbb{Q}}(t)$ with $\mathcal{P}_{f}(t)=\mathcal{P}_{\bar{f}}(h(t))$. Taking derivatives in the first component of the latter equation, and comparing it with the second, one has

$$
f^{\prime}(t)=\bar{f}^{\prime}(h(t)) h^{\prime}(t)=\bar{f}^{\prime}(h(t))
$$


Since $f(t)$ is not constant, $h(t)=t+c$ for some $c \in \overline{\mathbb{Q}}$. As $[\mathbb{Q}(\alpha): \mathbb{Q}]=2$, one can write $f(t)=\left(a_{n}+b_{n} \alpha\right) t^{n}+\alpha q_{1}(t)+q_{2}(t)$ and $\bar{f}(t)=\left(a_{n}+b_{n} \bar{\alpha}\right) t^{n}+\bar{\alpha} q_{3}(t)+q_{4}(t)$, for some $n \geq 0$ and some $q_{1}, q_{2}, q_{3}, q_{4} \in \mathbb{Q}[t]$ with $\operatorname{deg}\left(q_{j}\right) \leq n-1, a_{n}, b_{n} \in \mathbb{Q}$. So $a_{n}+b_{n} \alpha=a_{n}+b_{n} \bar{\alpha}$. On one hand, if $b_{n} \neq 0$, then $\alpha=\bar{\alpha}$ and $f(t) \in \mathbb{Q}[t]$. On the other hand, if $b_{n}=0$, then $f(t)=a_{n} t^{n}+\left(a_{n-1}+\alpha b_{n-1}\right) t^{n-1}+\alpha q_{5}(t)+q_{6}(t)$, and one can recursively deduce the result. If we end up with $b_{0}=b_{1}=\cdots=b_{n}=0$, then we have $f(t) \in \mathbb{Q}[t]$.

If $f(t)$ is not a polynomial, the proof goes as in the proof of Theorem 6 in [3] or Theorem 3.14 in [4].

The following corollary follows from the reasoning in the proof of the previous theorem.

Corollary 4.11. Let $f(x) \in \mathbb{Q}(\alpha)(x)$, with $[\mathbb{Q}(\alpha): \mathbb{Q}] \leq 2$, be a rational solution of $\mathcal{S}$. It holds that

1. If $f(t) \in \mathbb{Q}(\alpha)[t]$, then $f(t) \in \mathbb{Q}[t]$.

2. If

$$
f(t)=\frac{\alpha p_{1}(t)+p_{2}(t)}{t^{m}+a_{m-1} t^{m-1}+\cdots+a_{0}} \in \mathbb{Q}(\alpha)(t) \backslash \mathbb{Q}(\alpha)[t],
$$

where $p_{1}, p_{2} \in \mathbb{Q}[t]$ and $a_{i} \in \mathbb{Q}(\alpha)$, then

$$
f\left(t-\frac{a_{m-1}}{m}\right) \in \mathbb{Q}(t) .
$$

\section{$5 \quad$ Algorithms and Examples}

In this section, we outline two different algorithms derived from the previous ideas and we illustrate them by some examples. Finally, we compare them. The first algorithm assumes that a rational proper parametrization of the initial algebraic system of AODEs is known while the second either computes a parametrization or proposes a candidate of parametrization that has to be checked. Any of these approaches provide all the rational solutions (if they exist) of the system; note that, a parametrization, being solution of the differential system, exists if and only if the candidate parametrization turns out to be a solution.

We start with the general algorithm, where the main steps are modularized in sub-algorithms.

\section{Algorithm 1}

Input: an algebraic system of AODEs $\mathcal{S}$, associated to the set of polynomials $\mathcal{F}$ describing a curve $\mathcal{C} ; \mathcal{S}$ is assumed to satisfy the hypotheses described in Section 2. A rational proper parametrization $\mathcal{R}(t)=\left(r_{1}(t), r_{2}(t), \ldots, r_{n+1}(t)\right)$ of $\mathcal{C}$ with coefficients in a finite field extension $\mathbb{Q}(\alpha)$ of $\mathbb{Q}$ of degree at most 2 . 
Output: Decision on the existence of a nontrivial rational solution of $\mathcal{S}$, and, in the positive case, a rational general solution of $\mathcal{S}$ expressed over $\mathbb{Q}$.

\section{1. [Degree conditions]}

1.1. Apply Theorem 3.2 to compute $\operatorname{deg}_{\left\{w_{0}, w_{1}, \ldots, w_{n}\right\} \backslash\left\{w_{i}\right\}}(\mathcal{C})$ for $i=0,1, \ldots, n$.

1.2. Check whether the inequalities, $i=0,1, \ldots, n-1$,

$$
\operatorname{deg}_{\left\{w_{0}, w_{1}, \ldots, w_{n}\right\} \backslash\left\{w_{i}\right\}}(\mathcal{C})-1 \leq \operatorname{deg}_{\left\{w_{0}, w_{1}, \ldots, w_{n}\right\} \backslash\left\{w_{i+1}\right\}}(\mathcal{C}) \leq 2 \operatorname{deg}_{\left\{w_{0}, w_{1}, \ldots, w_{n}\right\} \backslash\left\{w_{i}\right\}}(\mathcal{C}) .
$$

hold. If not Return $\ll \mathcal{S}$ does not have nontrivial rational solutions 》.

\section{2. [Check of solutions]}

2.1. Let $A:=\frac{r_{2}(t)}{r_{1}^{\prime}(t)}$. If $A$ is neither a constant nor a quadratic polynomial with a double root, then Return $\ll \mathcal{S}$ does not have nontrivial rational solutions $\gg$.

2.2. If for some $\{2, \ldots, n+1\}, A(t) r_{j}^{\prime}(t) \neq r_{j+1}(t)$ Return $\ll \mathcal{S}$ does not have nontrivial rational solutions $\gg$.

2.3. If $A$ is constant then compute $f(x):=r_{1}(A x)$ else if $A=a(t-b)^{2}$ then compute $f(x)=r_{1}\left(\frac{a b x-1}{a x}\right)$.

2.4. If $f(x)$ is a polynomial,

Return $\ll$ a rational general solution of $\mathcal{S}$ is $f(x+C) \gg$

else

express $f(x)$ as

$$
\frac{\alpha p_{1}(x)+p_{2}(x)}{x^{m}+a_{m-1} x^{m-1}+\cdots+a_{0}} \in \mathbb{Q}(\alpha)(x) \backslash \mathbb{Q}(\alpha)[x],
$$

where $p_{1}, p_{2} \in \mathbb{Q}[x]$ and $a_{i} \in \mathbb{Q}(\alpha)$.

Return $\ll$ a rational general solution of $\mathcal{S}$ is $f\left(x-\frac{a_{m-1}}{m}+C\right) \in \mathbb{Q}(x) \gg$.

We illustrate Algorithm 1 by some examples.

Example 5.1. We consider the system of ODEs given by

$$
\mathcal{S}=\left\{\begin{array}{c}
\left(y^{\prime}(x)\right)^{2}-y^{\prime \prime}(x)=0 \\
y^{\prime}(x)-y(x)=0
\end{array}\right\} .
$$

It is clear that $\operatorname{deg}_{\left\{w_{1}, w_{2}\right\}}(\mathcal{C})=1, \operatorname{deg}_{\left\{w_{0}, w_{2}\right\}}(\mathcal{C})=1$ and $\operatorname{deg}_{\left\{w_{0}, w_{1}\right\}}(\mathcal{C})=3$. The degree conditions are not satisfied. One can conclude that $\mathcal{S}$ does not have nontrivial rational solutions. 
Example 5.2. Let $a, b, c, d \in \mathbb{C}$ such that $a d-c b \neq 0$. We consider the family of systems of ODEs given by

$$
\mathcal{S}=\left\{\begin{array}{l}
4 c^{2} y(x) y^{\prime}(x)^{3}-a d y(x) y^{\prime \prime}(x)^{2}+b c y(x) y^{\prime \prime}(x)^{2}+c y^{\prime \prime}(x) y(x)-2 c y^{\prime}(x)^{2}-a y^{\prime \prime}(x)=0 \\
c y^{\prime \prime}(x) y(x)-2 c y^{\prime}(x)^{2}-a y^{\prime \prime}(x)=0 \\
2 c^{2} y(x) y^{\prime}(x)-2 c a y^{\prime}(x)-a d y^{\prime \prime}(x)+b c y^{\prime \prime}(x)=0 \\
4 c^{2} y(x) y^{\prime}(x)^{3} y^{\prime \prime}(x)-a d y(x) y^{\prime \prime}(x)^{3}+b c y(x) y^{\prime \prime}(x)^{3}+c^{2} y(x)^{2}+c y(x) y^{\prime \prime}(x)^{2} \\
-2 c y^{\prime}(x)^{2} y^{\prime \prime}(x)-2 c a y(x)-a d y^{\prime}(x)-a y^{\prime \prime}(x)^{2}+b c y^{\prime}(x)+a^{2}=0
\end{array}\right\} .
$$

The corresponding associated algebraic system is given by

$$
\mathcal{F}=\left\{\begin{array}{l}
4 c^{2} w_{0} w_{1}^{3}-a d w_{0} w_{2}^{2}+b c w_{0} w_{2}^{2}+c w_{2} w_{0}-2 c w_{1}^{2}-a w_{2} \\
c w_{2} w_{0}-2 c w_{1}^{2}-a w_{2} \\
2 c^{2} w_{0} w_{1}-2 c a w_{1}-a d w_{2}+b c w_{2} \\
4 c^{2} w_{0} w_{1}^{3} w_{2}-a d w_{0} w_{2}^{3}+b c w_{0} w_{2}^{3}+c^{2} w_{0}^{2}+c w_{0} w_{2}^{2} \\
-2 c w_{1}^{2} w_{2}-2 c a w_{0}-a d w_{1}-a w_{2}^{2}+b c w_{1}+a^{2}
\end{array}\right\}
$$

The variety defined by $\mathcal{F}$ over $\overline{\mathbb{Q}}$ has dimension 1 , and it is a rational curve, say $\mathcal{C}$. Indeed a rational proper parametrization $\mathcal{R}(t):=\left(r_{1}(t), r_{2}(t), r_{3}(t), r_{4}(t)\right)$ of $\mathcal{C}$ is

$$
\mathcal{R}(t)=\left(\frac{1}{2} \frac{2 a c+(a d-c b) t}{c^{2}}, \frac{1}{4} \frac{t^{2}(a d-c b)}{c^{2}}, \frac{1}{4} \frac{t^{3}(a d-c b)}{c^{2}}\right) .
$$

Applying Theorem 3.2, one gets that $\operatorname{deg}_{\left\{w_{1}, w_{2}\right\}}(\mathcal{C})=1, \operatorname{deg}_{\left\{w_{0}, w_{2}\right\}}(\mathcal{C})=2$ and $\operatorname{deg}_{\left\{w_{0}, w_{1}\right\}}(\mathcal{C})=3$. So, the degree conditions do not exclude the existence of nontrivial solutions. Moreover, one has

$$
\frac{r_{2}(t)}{r_{1}^{\prime}(t)}=\frac{r_{3}(t)}{r_{2}^{\prime}(t)}=\frac{t^{2}}{2}
$$

So $\mathcal{S}$ has rational solutions, and a rational general solution of $\mathcal{S}$ is

$$
f(x):=r_{1}\left(\frac{-2}{x+C}\right)=\frac{(c b-a d)+a c(x+C)}{c^{2}(x+C)},
$$

for $C \in \mathbb{C}$. One can easily check $y(x)$ satisfies the equations in $\mathcal{S}$ by substitution.

In the particular case of $a d=c b$, if $c=0$, from the equations in $\mathcal{S}$ one has $a=0$ and the system disappears. On the other hand, if $c \neq 0$, the system $\mathcal{S}$ is easily solved. Its solution is the trivial one given by $f(x)=\frac{-a^{2}}{c^{2}-2 c a}$ in the case that $c \neq 2 a$; and the problem does not admit a solution if $c=2 a$.

Example 5.3. Let $a, b, c, d \in \mathbb{Q}$ such that $a d-c b \neq 0$ and $c \neq 0$. We consider the 
following family of systems of algebraic ODEs

$$
\mathcal{S}=\left\{\begin{array}{l}
27 y(x) y^{\prime \prime}(x)^{4} c^{2}-2 a d y(x) y^{\prime \prime \prime}(x)^{3}+2 b c y(x) y^{\prime \prime \prime}(x)^{3}+2 y^{\prime}(x) y^{\prime \prime \prime}(x) \\
-3 y^{\prime \prime}(x)^{2}=0 \\
2 y^{\prime}(x) y^{\prime \prime \prime}(x)-3 y^{\prime \prime}(x)^{2}=0 \\
9 y(x) y^{\prime}(x) y^{\prime \prime}(x)^{2} c^{2}-a d y(x) y^{\prime \prime \prime}(x)^{2}+b c y(x) y^{\prime \prime \prime}(x)^{2}+2 y^{\prime}(x) y^{\prime \prime \prime}(x) \\
-3 y^{\prime \prime}(x)^{2}=0 \\
27 c^{2} y^{\prime \prime}(x)^{5}-2 a d y^{\prime \prime}(x) y^{\prime \prime \prime}(x)^{3}+2 b c y^{\prime \prime}(x) y^{\prime \prime \prime}(x)^{3}+6 c^{2} y^{\prime}(x)^{2}-a d y^{\prime \prime \prime}(x) \\
+b c y^{\prime \prime \prime}(x)=0 \\
c y^{\prime \prime \prime}(x) y(x)-3 c y^{\prime}(x) y^{\prime \prime}(x)-a y^{\prime \prime \prime}(x)=0 \\
3 y(x) c^{2} y^{\prime \prime}(x)-3 a c y^{\prime \prime}(x)-a d y^{\prime \prime \prime}(x) \\
+b c y^{\prime \prime \prime}(x)=0 \\
2 c^{2} y(x) y^{\prime}(x)-2 a c y^{\prime}(x)-a d y^{\prime \prime}(x)+b c y^{\prime \prime}(x)=0 \\
c^{2} y(x)^{2}-2 c a y(x)-a d y^{\prime}(x)+b c y^{\prime}(x)+a^{2}+2 y^{\prime}(x) y^{\prime \prime \prime}(x)-3 y^{\prime \prime}(x)^{2}=0
\end{array}\right\} .
$$

The corresponding associated algebraic system is given by the polynomials

$$
\mathcal{F}=\left\{\begin{array}{l}
27 w_{0} w_{2}^{4} c^{2}-2 a d w_{0} w_{3}^{3}+2 b c w_{0} w_{3}^{3}+2 w_{1} w_{3}-3 w_{2}^{2}, \\
2 w_{1} w_{3}-3 w_{2}^{2}, 9 w_{0} w_{1} w_{2}^{2} c^{2}-a d w_{0} w_{3}^{2}+b c w_{0} w_{3}^{2}+2 w_{1} w_{3}-3 w_{2}^{2}, \\
27 c^{2} w_{2}^{5}-2 a d w_{2} w_{3}^{3}+2 b c w_{2} w_{3}^{3}+6 c^{2} w_{1}^{2}-a d w_{3}+b c w_{3} \\
c w_{3} w_{0}-3 c w_{1} w_{2}-a w_{3}, \\
3 w_{0} c^{2} w_{2}-3 a c w_{2}-a d w_{3}+b c w_{3}, \\
2 c^{2} w_{0} w_{1}-2 a c w_{1}-a d w_{2}+b c w_{2}, \\
c^{2} w_{0}^{2}-2 c a w_{0}-a d w_{1}+b c w_{1}+a^{2}+2 w_{1} w_{3}-3 w_{2}^{2}
\end{array}\right\} .
$$

The variety defined by $\mathcal{F}$ over $\overline{\mathbb{Q}}$ has dimension 1 , and it is a rational curve, say $\mathcal{C}$. Indeed, a rational proper parametrization $\mathcal{R}(t):=\left(r_{1}(t), r_{2}(t), r_{3}(t), r_{4}(t)\right)$ of $\mathcal{C}$, is

$$
\mathcal{R}(t)=\left(\frac{a c t+c b-a d}{c^{2} t}, \frac{a d-c b}{c^{2} t^{2}}, \frac{2(c b-a d)}{c^{2} t^{3}}, \frac{6(a d-c b)}{c^{2} t^{4}}\right) .
$$

Applying Theorem 3.2, one gets that $\operatorname{deg}_{\left\{w_{1}, w_{2}, w_{3}\right\}}(\mathcal{C})=1, \operatorname{deg}_{\left\{w_{0}, w_{2}, w_{3}\right\}}(\mathcal{C})=2$, $\operatorname{deg}_{\left\{w_{0}, w_{1}, w_{3}\right\}}(\mathcal{C})=3$ and $\operatorname{deg}_{\left\{w_{0}, w_{1}, w_{2}\right\}}(\mathcal{C})=4$. So, again, the degree conditions do not exclude the existence of nontrivial solutions. Moreover

$$
\frac{r_{4}(t)}{r_{3}^{\prime}(t)}=\frac{r_{3}(t)}{r_{2}^{\prime}(t)}=\frac{r_{2}(t)}{r_{1}^{\prime}(t)}=1 .
$$

So $\mathcal{S}$ has rational solutions, and a rational general solution of $\mathcal{S}$ is

$$
f(x):=r_{1}(x+C)=\frac{a c(x+C)+c b-a d}{c^{2}(x+C)},
$$

for $C \in \mathbb{C}$. It is easily checked that, if $c b=a d$, then the system is reduced to the equation $2 y^{\prime}(x) y^{\prime \prime \prime}(x)-3 y^{\prime \prime}(x)^{2}=0$ in the case that $c=0$, so it does not fit into the systems under study in this work. On the other hand, if $c \neq 0$ the only solution of $\mathcal{S}$ is the trivial solution $f(x)=\frac{a}{c}$. 
Example 5.4. We consider the system

$$
\mathcal{S}=\left\{\begin{array}{l}
y(x)^{2}-y^{\prime}(x)=0 \\
y(x)^{3}-y^{\prime \prime}(x)=0
\end{array}\right\}
$$

The associated curve $\mathcal{C}$ can be properly parametrized as $\mathcal{R}(t):=\left(t, t^{2}, t^{3}\right)$. $\operatorname{deg}_{\left\{w_{0}, w_{1}\right\}}(\mathcal{C})=3, \operatorname{deg}_{\left\{w_{0}, w_{2}\right\}}(\mathcal{C})=2, \operatorname{deg}_{\left\{w_{1}, w_{2}\right\}}(\mathcal{C})=1$. So the degree conditions are satisfied. It is immediate to check that $\frac{r_{3}(t)}{r_{2}^{\prime}(t)} \neq \frac{r_{2}(t)}{r_{1}^{\prime}(t)}$, so that there does not exist a non-constant rational solution of $\mathcal{S}$. Indeed, the general solution of $\mathcal{S}$ is $y(x)=0$ : differentiating the first equation one arrives at

$$
2 y(x) y^{\prime}(x)-y^{\prime \prime}(x)=0
$$

In addition, from the second equation, we have $y^{\prime \prime}(x)=y^{3}(x)$, and by substitution in (6), we get $2 y(x) y^{\prime}(x)-y^{3}(x)=0$. This yields $y(x)=0$ or $2 y^{\prime}(x)=y^{2}(x)$. The first equation in $\mathcal{S}$ and $2 y^{\prime}(x)=y^{2}(x)$ yield $y(x)=0$.

Our second algorithm reduces the study of the initial system $\mathcal{S}$ to the study of a system $\mathcal{S}^{\prime}$,which shares the same behavior with respect to the existence of rational solutions. Moreover, one can find an equation in $\mathcal{S}^{\prime}$, say $F=0$, only depending on $y$ and $y^{\prime}$ so that one can apply the results in [3] to that equation, and complete this information with the other equations involved in $\mathcal{S}^{\prime}$.

\section{Algorithm 2.}

Input: an algebraic system of AODEs $\mathcal{S}$, associated to the set of polynomials $\mathcal{F}$ describing a curve $\mathcal{C} ; \mathcal{S}$ is assumed to satisfy the hypotheses described in Section 2 .

Output: Decision on the existence of a nontrivial rational solution of $\mathcal{S}$, and, in the positive case, a rational general solution of $\mathcal{S}$ expressed over $\mathbb{Q}$.

1. Apply Step 1 (Degree conditions) of Algorithm 1. For computing $\operatorname{deg}_{\left\{w_{0}, w_{1}, \ldots, w_{n}\right\} \backslash\left\{w_{i}\right\}}(\mathcal{C})$ for $i=0,1, \ldots, n$, one can take a random hyperplane $\mathcal{H}$ in $\mathbb{Q}^{n+1}$, of the form $w_{i}=\lambda$, and compute the number of intersections of $\mathcal{H}$ with $\mathcal{C}$. This probabilistic method could be made deterministic by the computation of a comprehensive Gröbner basis for $\mathcal{F} \cup\left\{w_{i}=\lambda\right\}$, from which the general number of intersections with such hyperplanes can be determined.

\section{2. [Projected curve computation]}

2.1. Compute a Gröbner basis for $\mathcal{F}$ w.r.t. the lex order, with $w_{n}>w_{n-1}>$ $\ldots>w_{1}>w_{0}$, say $\mathcal{F}_{G}$; reduced in the sense of Def. 5 in [1], pg. 90. For $j=0, \ldots, n$, let $\mathcal{F}_{j}$ be the elements in the previous Gröbner basis which depend on $\left\{w_{0}, w_{1}, \ldots, w_{j}\right\}$ and $w_{j}$ appears. If $\mathcal{F}_{0} \neq \emptyset$ then Return $\ll \mathcal{S}$ does not have nontrivial rational solutions $\gg$. 
2.2. Compute a proper rational parametrization $\mathcal{R}(t)=\left(r_{1}(t), r_{2}(t)\right)$ of $\mathcal{C}$ (i.e. the curve defined by $\mathcal{F}_{1}$; that is by the gcd of all polynomials in $\mathcal{F}_{1}$ ) with coefficients in a finite field extension of $\mathbb{Q}$ of degree at most 2. For this, apply the algorithms in [11]. If there exists $r_{j}(t)$, for $j=1,2$, with at least two different simple poles, one can apply Corollary 4.4 , then Return $\ll \mathcal{S}$ does not have nontrivial rational solutions $\gg$.

3. [Curve lift and check of solutions]

3.1. Let $A:=\frac{r_{2}(t)}{r_{1}^{\prime}(t)}$. If $A$ is neither a constant nor a quadratic polynomial with a double root, Return $\ll \mathcal{S}$ does not have nontrivial rational solutions $\gg$.

3.2. If $A$ is constant then compute $f(x):=r_{1}(A x)$ else if $A=a(t-b)^{2}$ then compute $f(x)=r_{1}\left(\frac{a b x-1}{a x}\right)$.

If $A$ is constant set $r_{i}(t)=f^{(i-1)}(t)$ for $i=3, \ldots, n+1$ and go to Step 3.3.3. of Option-2.

Option 1: Checking the candidate for solution by derivation

3.3. Compute $f^{\prime}(x)$. For $i=2, \ldots, n$ do

3.3.1. Compute $f^{(i)}$.

3.3.2. If $F\left(f(x), f^{\prime}(x), f^{\prime \prime}(x), \ldots, f^{(i)}(x)\right) \neq 0$ for some $F \in \mathcal{F}_{i}$, then Return $\ll$ $\mathcal{S}$ does not have nontrivial rational solutions $\gg$.

3.4. Apply Step 2.4. of Algorithm 1.

Option 2: Checking the candidate for solution by lifting $\mathcal{R}(t)$

3.3. For $i=3, \ldots, n$ do

3.3.1. Compute $M\left(t, w_{i-1}\right):=\operatorname{gcd}_{\overline{\mathbb{Q}}(t)\left[w_{i-1}\right]}\left(F\left(r_{1}(t), \ldots, r_{i-1}(t), w_{i-1}\right) \mid F \in\right.$ $\left.\mathcal{F}_{i-1}\right\}$.

3.3.2. Let $r_{i}(t)$ be the only root of $M\left(t, w_{i-1}\right)$ in $\overline{\mathbb{Q}}(t)$.

3.3.3. If $A(t) r_{i-1}^{\prime}(t) \neq r_{i}(t)$ Return $\ll \mathcal{S}$ does not have nontrivial rational solutions $\gg$.

3.4. There exist rational solutions and for finding them apply Step 2.4. of Algorithm 1.

Remark 5.5. In Step 2.1, if $\mathcal{F}_{0}$ is not empty, we return that there does not exist a nontrivial rational solution. The reason is the following. If $\mathcal{F}_{0} \neq \emptyset$ then the first component of the parametrization $\mathcal{R}(t)$ is a constant $\lambda$, and therefore any rational solution would be $(\lambda, 0, \ldots, 0)$ and hence constant.

We illustrate Algorithm 2 by some examples. 
Example 5.6. We consider the system provided in Example 5.2. We directly go to Step 2 of Algorithm 2, since we already know from Example 5.2 that the degree conditions are satisfied. In Step 2 we get

$$
\begin{aligned}
\mathcal{F}_{G}= & \left\{-c^{2} w_{0}^{2}+2 c a w_{0}+(a d-b c) w_{1}-a^{2},\right. \\
& \left.-2 c^{4} w_{0}^{3}+6 a c^{3} w_{0}^{2}-6 a^{2} c^{2} w_{0}+\left(a^{2} d^{2}-2 a b c d+b^{2} c^{2}\right) w_{2}+2 a^{3} c\right\}
\end{aligned}
$$

So

$$
\begin{aligned}
& \mathcal{F}_{1}=\left\{-c^{2} w_{0}^{2}+2 c a w_{0}+(a d-b c) w_{1}-a^{2}\right\} \\
& \mathcal{F}_{2}=\left\{-2 c^{4} w_{0}^{3}+6 a c^{3} w_{0}^{2}-6 a^{2} c^{2} w_{0}+\left(a^{2} d^{2}-2 a b c d+b^{2} c^{2}\right) w_{2}+2 a^{3} c\right\}
\end{aligned}
$$

We get

$$
\mathcal{R}(t)=\left(r_{1}(t), r_{2}(t)\right)=\left(\frac{1}{2} \frac{2 a c+(a d-c b) t}{c^{2}}, \frac{1}{4} \frac{t^{2}(a d-c b)}{c^{2}}\right)
$$

as a proper parametrization of the curve $\mathcal{C}$ defined by $-c^{2} w_{0}^{2}+2 c a w_{0}+(a d-b c) w_{1}-$ $a^{2}=0$. In Step 3.1. we get that $A(t)=\frac{1}{2} t^{2}$, and in Step 3.2.

$$
f(x)=r_{1}\left(\frac{2}{x}\right)=\frac{c a x+a d-b c}{x c^{2}} .
$$

1. Option-1. We compute $f^{\prime}(x)=(a d-b c) /\left(x^{2} c^{2}\right), f^{\prime \prime}(x)=-2(a d-b c) /\left(x^{3} c^{2}\right)$ and we check that for all $F \in \mathcal{F}_{2}$ we have $F\left(f, f^{\prime}, f^{\prime \prime}\right)=0$.

2. Option-2. Since $\mathcal{F}_{2}$ contains only one polynomial, say $F\left(w_{0}, w_{1}, w_{2}\right)$, we compute the root of $F\left(r_{1}(t), r_{2}(t), w_{2}\right)$ w.r.t. $w_{2}$, namely

$$
r_{3}(t)=\frac{(a d-b c) t^{3}}{4 c^{2}} .
$$

We check that $A(t) r_{2}^{\prime}(t)=r_{3}(t)$.

Applying Step 2.4. of Algorithm 1 we get that the rational solutions are $f(x+C)$.

Example 5.7. We consider the system provided in Example 5.3. We directly go to Step 2 of Algorithm 1, since we already know from Example 5.3 that the degree conditions are satisfied. In Step 2 we get the Gröbner basis given by

$$
\begin{aligned}
\mathcal{F}_{G}= & \left\{-c^{2} w_{0}^{2}+2 c a w_{0}+a d w_{1}-b c w_{1}-a^{2}\right. \\
& -2 c^{4} w_{0}^{3}+6 a c^{3} w_{0}^{2}-6 a^{2} w_{0} c^{2}+a^{2} d^{2} w_{2}-2 a b c d w_{2}+b^{2} c^{2} w_{2}+2 a^{3} c \\
& -6 c^{6} w_{0}^{4}+24 c^{5} w_{0}^{3} a-36 a^{2} c^{4} w_{0}^{2}+24 a^{3} w_{0} c^{3}+w_{3} a^{3} d^{3}-3 w_{3} a^{2} d^{2} b c+3 c^{2} w_{3} a d b^{2} \\
& \left.-w_{3} b^{3} c^{3}-6 a^{4} c^{2}\right\}
\end{aligned}
$$

So

$$
\begin{aligned}
\mathcal{F}_{1}= & \left\{-c^{2} w_{0}^{2}+2 c a w_{0}+a d w_{1}-b c w_{1}-a^{2}\right\} \\
\mathcal{F}_{2}= & \left\{-2 c^{4} w_{0}^{3}+6 a c^{3} w_{0}^{2}-6 a^{2} w_{0} c^{2}+a^{2} d^{2} w_{2}-2 a b c d w_{2}+b^{2} c^{2} w_{2}+2 a^{3} c\right\} \\
\mathcal{F}_{3}= & \left\{-6 c^{6} w_{0}^{4}+24 c^{5} w_{0}^{3} a-36 a^{2} c^{4} w_{0}^{2}+24 a^{3} w_{0} c^{3}+w_{3} a^{3} d^{3}-3 w_{3} a^{2} d^{2} b c+3 c^{2} w_{3} a d b^{2}\right. \\
& \left.-w_{3} b^{3} c^{3}-6 a^{4} c^{2}\right\}
\end{aligned}
$$


We get

$$
\mathcal{R}(t)=\left(r_{1}(t), r_{2}(t)\right)=\left(\frac{a c t+c b-a d}{c^{2} t}, \frac{a d-c b}{c^{2} t^{2}}\right)
$$

as a proper parametrization of the curve $\mathcal{C}$ defined by $-c^{2} w_{0}^{2}+2 c a w_{0}+a d w_{1}-b c w_{1}-a^{2}=$ 0. In Step 3.1. we get that

1. Option-1. We compute $f^{\prime}(x)=(a d-b c) /\left(c^{2} x^{2}\right), f^{\prime \prime}(x)=-2(a d-b c) /\left(c^{2} x^{3}\right)$, and we check that for all $F \in \mathcal{F}_{2}$ we get that $F\left(f, f^{\prime}, f^{\prime \prime}\right)=0$. Afterwards, we compute $f^{\prime \prime \prime}(x)=6(a d-b c) /\left(c^{2} x^{4}\right)$ and we check that for all $F \in \mathcal{F}_{3}$ we have $F\left(f, f^{\prime}, f^{\prime \prime}, f^{\prime \prime \prime}\right)=0$.

2. Option-2. Since $\mathcal{F}_{2}$ has only one polynomial, say $F\left(v, w_{1}, w_{2}\right)$, we compute the root of $F\left(r_{1}(t), r_{2}(t), w_{2}\right)$ w.r.t. $w_{2}$, namely $r_{3}(t)=-2(a d-b c) /\left(c^{2} t^{3}\right)$. We check that $A(t) r_{2}^{\prime}(t)=r_{3}(t)$. Similarly for $\mathcal{F}_{3}$ we get $r_{4}(t)=6(a d-b c) /\left(c^{2} t^{4}\right)$ and that $A(t) r_{3}^{\prime}(t)=r_{4}(t)$.

Applying Step 2.4. of Algorithm 1 we get that the rational solutions are $f(x+C)$.

Example 5.8. In Example 5.4, one can check that $\mathcal{F}_{1}=\left\{w_{0}^{2}-w_{1}\right\}$. A parametrization for the curve $w_{0}^{2}-w_{1}=0$ is given by $\mathcal{R}(t)=\left(r_{1}(t), r_{2}(t)\right)=\left(t, t^{2}\right)$ which verifies that $r_{2}(t) / r_{1}(t)=t^{2}$. Following Algorithm 2, one is tempted to choose $f(x)=r_{1}(-1 / x)=$ $-1 / x$ as a solution of the system. However, it does not provide a solution for $\mathcal{F}_{2}=$ $\left\{w_{0}^{3}-w_{2}\right\}$, so there does not exist a rational solution of $\mathcal{S}$.

Example 5.9. Let $m \in \mathbb{N}, m \geq 2$. We consider the system of ODEs given by

$$
\mathcal{S}=\left\{\begin{array}{l}
y(x)^{2}-y^{\prime}(x)=0 \\
2 y(x)^{3}-y^{\prime \prime}(x)=0 \\
6 y(x)^{3}-y^{\prime \prime}(x)=0 \\
\vdots \\
m ! y(x)^{m+1}-y^{(m)}(x)=0 \\
y(x)^{m+2}-y^{(m+1)}(x)=0
\end{array}\right\}
$$

The corresponding associated algebraic system is given by

$$
\mathcal{F}=\left\{\begin{array}{l}
w_{0}^{2}-w_{1}=0 \\
2 w_{0}^{3}-w_{2}=0 \\
6 w_{0}^{3}-w_{3}=0 \\
\vdots \\
m ! w_{0}^{m+1}-w_{m+1}=0 \\
w_{0}^{m+2}-w_{m+2}=0
\end{array}\right\} .
$$

It is clear that $\operatorname{deg}_{\left\{w_{0}, w_{1}, \ldots, w_{m+2}\right\} \backslash\left\{w_{j}\right\}}(\mathcal{C})=j+1$ for every $j=0,1, \ldots, m+1$ and hence the degree conditions are satisfied. One has $\mathcal{R}(t)=\left(r_{1}(t), \ldots, r_{m+2}(t)\right)$ is a 
rational parametrization given by $r_{1}(t)=t, r_{j}(t)=(j-1) ! t^{j}$ for $j=1, \ldots, m+1$, and $r_{m+2}(t)=t^{m+2}$. It is clear that

$$
\frac{r_{2}(t)}{r_{1}^{\prime}(t)}=t^{2} \neq \frac{t^{2}}{(m+1) !}=\frac{r_{m+2}(t)}{r_{m+1}^{\prime}(t)} .
$$

At Step 2.2., Algorithm 1 concludes the nonexistence of a nontrivial rational solution of $\mathcal{S}$. On the other hand, Algorithm 2, either through Option 1 or 2, will need to execute all iterations of the loop till the last one (i.e. $\mathcal{O}(m))$.

We finish this section with a comparison of the two algorithms.

- The main advantage of Algorithm 1 is that the parametrization is provided and hence one does not have to go through the Gröbner basis computation, and lifting process. This enables the algorithm to directly check the existence conditions of rational solutions stated in Theorem 4.2. This can be clearly seen in Example 5.9. The main disadvantage is that the method is only applicable when a parametrization is easily deduced or when the parametrization is provided by the problem itself.

- In the general case, Algorithm 2 is more feasible than Algorithm 1. Within Algorithm 2 two options are considered. The first option computes, from the plane parametrization $\left(r_{1}(t), r_{2}(t)\right)$, the function $f(x)=r_{1}(\psi(x))$ where $\psi$ is a linear rational function. From there a candidate of parametrization is proposed, namely $\mathcal{T}(x)=\left(f(x), f^{\prime}(x), \ldots, f^{(n)}(x)\right)$. This candidate is checked iteratively using the different elimination ideals provided by the Gröbner basis. The second option lifts the planar parametrization $\left(r_{1}(t), r_{2}(t)\right)$ to a parametrization $\left(r_{1}(t), \ldots, r_{n+1}(t)\right)$, iteratively and using also the elimination ideals. At each level, the process checks the existence of rational solution by comparing the corresponding parametrization component with the previous one. Observe that at each level $\left(r_{1}(t), \ldots, r_{i+1}(t)\right) \circ \psi(x)=\left(f(x), f^{\prime}(x), \ldots, f^{(i)}(x)\right)$. So, the difference is in terms of complexity, depending on how dense $f(x)$ is, which is connected to the form of $r_{1}(t)$ and $\psi(x)$, and/or how dense the polynomials in the Gröbner basis are; recall that one has to substitute the candidate in the polynomials to check whether they vanish. In case of density, it will be better to work with $\left(r_{1}, \ldots, r_{n+1}\right)$, i.e. option 2 , and perform the substitution by $\psi(x)$ at the end of the process, otherwise option 1 will be more efficient.

- As a final remark, we observe that if the initial system $\mathcal{F}$ has a polynomial depending only on two consecutive variable, say $w_{i}$ and $w_{i+1}$, then one does not need to compute the Gröbner basis. Indeed, from $w_{i}$ and $w_{i+1}$ one gets $A$. If $A$ is not of the required form then there is no rational solution. Otherwise, we compute $f$ and from $f$, taking derivatives and, if necessary (i.e if the variables are not $\left.w_{0}, w_{1}\right)$, integrals, one gets a candidate of solution that it checked by substituting in the system. Observe that if any of the integrals is not a rational function then one deduces that no rational solution exists. 


\section{Conclusion}

We have shown that the solution method of Feng and Gao for autonomous AODEs of order 1 can be generalized to systems of autonomous AODEs of arbitrary order but algebro-geometric dimension 1. It remains to be investigated whether an analogous generalization is also possible for non-autonomous AODEs as considered by Ngô and Winkler [6].

\section{Acknowledgements:}

A. Lastra is partially supported by the project MTM2012-31439 of Ministerio de Ciencia e Innovacion, Spain; member of the Research Group ASYNACS (Ref. CCEE2011/R34).

J. R. Sendra is partially supported by the Spanish Ministerio de Economía y Competitividad under the project MTM2011-25816-C02-01; member of the Research Group ASYNACS (Ref. CCEE2011/R34).

L. X. C. Ngô is partially supported by the Vietnam Institute for Advanced Study in Mathematics (VIASM).

F. Winkler is partially supported by the Austrian Science Fund (FWF): W1214-N15, project DK11, and by the Spanish Ministerio de Economía y Competitividad under the project MTM2011-25816-C02-01.

\section{References}

[1] Cox D., Little J., O'Shea D.: Ideals, Varieties, and Algorithms (2nd ed.). SpringerVerlag, New York (1997)

[2] Eremenko A.: Rational solutions of first-order differential equations, Ann. Acad. Sci. Fenn., Math., v. 23, 181-190 (1998)

[3] Feng, R., Gao, X.: Rational general solutions of algebraic ordinary differential equations. Proc. ISSAC 2004, 155-162 (2004)

[4] Feng, R., Gao, X.: A polynomial time algorithm for finding rational general solutions of first order autonomous ODEs. Journal of Symbolic computation 41, 739-762 (2006)

[5] Harris J.: Algebraic Geometry. A First Course. Springer-Verlag (1995)

[6] Ngô, L.X.C., Winkler, F.: Rational general solutions of first order non-autonomous parametrizable ODEs. Journal of Symbolic Computation 45/12, 1426-1441 (2010)

[7] Ngô, L.X.C., Winkler, F.: Rational general solutions of parametrizable AODEs. Publ. Math. Debrecen 79 (2011), no. 3-4, 573-587. 
[8] Ngô, L.X.C., Winkler, F.: Rational general solutions of planar rational systems of autonomous ODEs. Journal of Symbolic Computation 46/10, 1173-1186 (2011)

[9] Huang, Y., Ngô, L.X.C., Winkler, F.: Rational general solutions of higher order algebraic ODEs. Journal of Systems Science and Complexity 26, 261-280 (2013)

[10] Sendra, J.R. and Winkler, F.: Tracing index of rational curve parametrization, Comp. Aided Geo. Design 18(8), 771-795 (2001)

[11] Sendra, J.R., Winkler, F., Pérez-Díaz, S.: Rational algebraic curves: A computer algebra approach. Algorithms and Computation in Mathematics, 22. Springer, Berlin (2008)

[12] Winkler F.: Polynomial Algorithms in Computer Algebra. Springer-Verlag, Wien New York (1996) 